\title{
Research on Site Selection and Capacity Determination of Mobile Energy Storage in Extreme Scenarios Considering the Behaviour of Decision
}

\author{
Shiyu Deng ${ }^{1}{ }^{*}$, Chang Liu ${ }^{1}$, Wenxia Liu ${ }^{1}$ and Zongqi Liu ${ }^{1}$ \\ ${ }^{1}$ North China Electric Power University, School of Electrical and Electronic Engineering, 102206 Beijing, China
}

\begin{abstract}
In order to ensure that the power supply can be restored quickly and efficiently under extreme conditions, an evaluation and decision-making method for mobile energy storage site selection and capacity planning considering the behaviour of decision makers is proposed. The prospect value is calculated based on the prospect theory to describe the bounded rationality and loss aversion of decision makers, and the attribute weight is calculated based on the relative closeness, and then the comprehensive value is obtained. Based on this, we can choose a mobile energy storage installation plan that is more in line with the psychology of decision makers.
\end{abstract}

\section{Introduction}

Vigorously developing renewable energy has become an important strategic consensus for all countries in the world to deal with environmental pollution and resource depletion. Energy storage technology is recognized as one of the most effective measures to solve the problem of renewable energy consumption and improve the safe and economic operation of the power system.

Due to the strong flexibility of the energy storage device, mobile energy storage can be dispatched to provide stable power support for lifeline loads in extreme disaster scenarios, and to minimize the power outage loss of important loads.

The literature [1] takes into account the uncertainty of load forecasting, configures energy storage with the goal of minimizing energy storage investment costs and operation and maintenance costs, and comprehensively considers the power purchase costs, operation and maintenance costs, switching costs, losses of the distribution network and load penalty fees to coordinate and optimize measures such as energy storage operation, demand side management, and network reconfiguration. The literature [2] proposed a two-stage scheduling framework based on pre-positioning and real-time allocation for mobile energy storage, which realized the improvement of resilience. The literature [3] comprehensively considers the line reinforcement cost, energy storage investment cost and load loss cost, and coordinates and optimizes the line reinforcement and energy storage configuration with the goal of minimizing the overall cost, and the two work together to improve the emergency response capability of the distribution network. Literature [4] proposed a mixed integer programming model to solve the problems of mobile energy storage dispatching and distribution system operation at different time scales, road and grid coupling, etc., to improve the resilience of the distribution network by optimizing the dynamic dispatch of mobile power sources. However, measures to improve the resilience of the grid are usually costly. In terms of the benefits of the distribution network, the existing methods only consider the effect of improving the resilience, without considering the investment margin and the economy under normal operation, which may lead to excessive investment. Therefore, it is necessary to propose a reasonable energy storage configuration method to achieve a balanced improvement of resilience and economy.

In summary, in order to improve the accuracy of the results, this paper introduces the prospect theory into the decision-making process of mobile energy storage selection and designation capacity planning. First, this paper establishes a comprehensive index system through the analysis of the power supply recovery capacity and economics of mobile energy storage; secondly, we calculate the importance of the index based on the relative discount, and normalize the calculation of the index weight; finally, the schemes are sorted and selected based on the prospect theory multi-index decision-making method.

\section{Index system construction}

The planning scheme of energy storage includes the capacity configuration and installation location of energy storage. The investment cost of energy storage can be obtained through the planning scheme, including the initial investment cost and the operation and maintenance cost. At the same time, the capacity configuration of energy storage can be embodied as a constraint in the

\footnotetext{
* Corresponding author: dengsy@ncepu.edu.cn
} 
operation of energy storage. The evaluation indicators of the plan include not only the investment cost, but also the operating results under the plan, that is, the economic benefits obtained from normal operation, and the ability for self-healing recovery.

\subsection{Economic indicators}

1) The investment cost of energy storage

The investment cost of mobile energy storage is mainly divided into two parts, The investment cost of mobile energy storage is mainly divided into two parts, namely the initial investment cost and the operation and maintenance cost. The initial investment cost refers to the total amount of capital invested at the beginning of the construction of the distributed energy storage system project. Operation and maintenance cost refers to the cost investment of energy storage system due to the equipment loss, aging or the need for maintenance or replacement after the operation parameters reach the scrap standard in the total service life.

$$
\begin{gathered}
M_{f}=C_{i n v}+C_{o p} \\
C_{i n v}=\sum_{j \in N} C_{i n v, j}=\sum_{j \in N} \eta_{n_{j}}\left(C_{i n v, j}^{P} P_{j}+C_{i n v, j}^{E} E_{j}+C_{i n v, j}^{F}\right) \\
C_{o p}=\sum_{j \in N} C_{o p, j}=\sum_{j \in N} \eta_{n_{j}} \sum_{i=1}^{n_{j}} C_{o p j, i} P_{j, i}
\end{gathered}
$$

In the formula, $C_{i n v}$ is the initial investment cost, and $C_{o p}$ is the operation and maintenance cost. $N$ represents the total number of installed energy storage; $n_{j}$ refers to the operating life of energy storage battery $j$; represents the capital recovery coefficient; $P$ and $E$ represent the power and capacity of energy storage respectively; $C_{i n v, j}^{P}$, $C_{i n v, j}^{E}, \quad C_{i n v, j}^{F}$ represent the unit power cost and unit capacity cost of energy storage $j$, respectively and fixed installation costs.

2) Benefits from the participation of energy storage in normal operation

The benefits of energy storage participating in normal operation are mainly obtained through peak shaving and valley filling.

$$
W=\sum_{1}^{365} \sum_{i=1}^{24}\left(P_{i, d i s}-P_{i, c h}\right) p_{i}
$$

\subsection{Resilience index}

The evaluation of the quality of the self-healing recovery plan is not only related to the degree of recovery of important loads, but also related to the network status and fault characteristics after the fault recovery. Therefore, the strength of recovery requires comprehensive consideration of multiple indicators. The resilience indicators constructed in this paper are:

1). Proportion of isolated nodes

$$
K_{\text {iso }}=\frac{N_{\text {iso }}}{N}
$$

In the formula, $N_{\text {iso }}$ represents the number of isolated nodes, and $N$ represents the total number of nodes.

2). Fault recovery time $T$

3). Switch operation times K

4). Loss of power load L

The power loss load is calculated according to the priority of the load, and the important load is restored first.

$$
L=\sum_{i \in N_{L}} \sum_{j \in N_{L i}} c_{i j} x_{i j} L_{i j}
$$

Among them, $N_{L}$ represents the number of power-loss nodes, $N_{L i}$ represents the number of loads on node $i, N K$ represents the number of switches, $c_{i j}$ is the importance of the $j$ th load in node $i$, and $x_{i j}$ represents the power supply status of the $j$ th load in node $i$, in which $x_{i j}=1$ means the load has regained power, and $x_{i j}=0$ means the load has failed to regain power.

\section{Decision and evaluation model of energy storage scheme based on Prospect Theory}

Prospect theory believes that decision-makers have limited rationality in actual decision-making [5-], and with changes in external factors, the preferences of decision-makers will also change. When gains appear, decision makers tend to avoid risks. At this time, the value function of decision makers is a concave function; when there is loss, decision makers often show a risk preference, and then the value function is convex. Among them, the gains and losses are relative values relative to the standard set by the decision maker.

\subsection{Prospect value function}

Based on different reference points, the prospect value of different solutions can be calculated, namely:

$$
V_{i}=\sum_{j=1}^{m} \pi\left(p_{j}\right) v\left(\Delta x_{j}\right)
$$

Among them, $V_{i}$ is the prospect value of the planning scheme $N i ; v\left(\Delta x_{j}\right)$ represents the prospect value function of the scheme $\mathrm{Ni}$ at the $j$ th attribute value and the reference point difference $\Delta x_{j} ; \pi$ represents the probability weight function, and its size represents the influence of the attribute weight $p_{i}$ on the entire foreground.

Where, the value function is as follow:

$$
v\left(\Delta x_{j}\right)=\left\{\begin{array}{c}
\left(\Delta x_{j}\right)^{\alpha}, \Delta x_{i}>0 \\
-\lambda\left(-\Delta x_{j}\right)^{\beta}, \Delta x_{i}<0
\end{array}\right.
$$

Among them, $\alpha$ and $\beta$ respectively represent the degree of unevenness of the value function relative to the gain and loss of the image, which measures the degree of 
preference of decision-makers for risk-taking, and $0<\alpha$, $\beta<1$; the larger the value of $\lambda(\lambda>1)$, the more averse the decision-maker is to loss, the more sensitive the decisionmaker is relative to the equivalent loss. A large number of experimental results show that the values of $\alpha=\beta=0.88$ and $\lambda=2.25$ are consistent with empirical data.

The probability weight functions when facing gains and losses are

$$
\begin{aligned}
& \pi(p)^{+}=\frac{p^{\chi}}{\left[p^{\chi}+(1-p)^{\chi}\right]^{1 / \chi}} \\
& \pi(p)^{-}=\frac{p^{\delta}}{\left[p^{\delta}+(1-p)^{\delta}\right]^{1 / \delta}}
\end{aligned}
$$

In the formula, $\pi^{+}(p)$ represents the non-linear probability weight formula of the return; $\pi^{-}(p)$ represents the non-linear probability weight formula at the time of loss, $\chi$ is the size of the risk when representing the return, and $\delta$ represents the risk attitude coefficient at the time of loss. The two coefficients indicate the degree of profit preference and risk aversion of the decision maker. A large number of experimental results show that $\chi=0.61, \delta=$ 0.69 is more consistent with the actual situation.

\subsection{Construction of Prospect Value Matrix}

There is an energy storage planning scheme set $\mathrm{N}=\left\{\mathrm{N}_{1}\right.$, $\left.\mathrm{N}_{2}, \ldots, \mathrm{N}_{\mathrm{n}}\right\}$. In this paper, each indicator is used as the attribute of the scheme, and the attribute set of each scheme is $A=\{A 1, A 2, \ldots, A m\}$, and then the effect vector of the planning scheme $N_{i}$ under the attribute set $\mathrm{A}$ is

$$
x_{i}=\left(x_{i 1}, x_{i 2}, \cdots, x_{i m}\right)
$$

The sample matrix of the effect of scheme set $\mathrm{N}$ on attribute set $\mathrm{A}$ is

$$
\begin{aligned}
X & =\left(x_{1}, x_{2}, \cdots, x_{n}\right)^{T} \\
& =\left[\begin{array}{cccc}
x_{11} & x_{12} & \ldots & x_{1 m} \\
x_{21} & x_{22} & \ldots & x_{2 m} \\
\ldots & \ldots & \ldots & \ldots \\
x_{n 1} & x_{n 2} & \ldots & x_{n m}
\end{array}\right]
\end{aligned}
$$

Normalize it as

$$
r_{i j}=\frac{x_{i j}-\max _{1 \leq i \leq n}\left\{x_{i j}\right\}}{\max _{1 \leq i \leq n}\left\{x_{i j}\right\}-\min _{1 \leq i \leq n}\left\{x_{i j}\right\}}
$$

The positive and negative ideal points are

$$
\begin{aligned}
& R^{+}=\left\{r_{1}^{+}, r_{2}^{+}, \cdots, r_{m}^{+}\right\} \\
& R^{-}=\left\{r_{1}^{-}, r_{2}^{-}, \cdots, r_{m}^{-}\right\}
\end{aligned}
$$

Where,

$$
\begin{aligned}
& r_{j}^{+}=\max _{1 \leq i \leq n}\left(r_{i j}\right) \\
& r_{j}^{-}=\min _{1 \leq i \leq n}\left(r_{i j}\right)
\end{aligned}
$$

Calculate the value function of each attribute according to the formula (8). Then calculate attribute weights based on relative closeness ${ }^{[6]}$ and the smaller the relative closeness, the greater its contribution. Attribute weight can be calculated as follows.

$$
\omega_{i}=\varphi_{i} / \sum_{i=1}^{n} \varphi_{i}
$$

According to the above, the prospect value of each program can be calculated. And according to the value of $V_{i}$ to sort the optimal scheme.

\section{Case analysis}

In order to comprehensively evaluate the resilience and economy of the mobile energy storage configuration plan, the overall idea of this article is shown in figure 1.

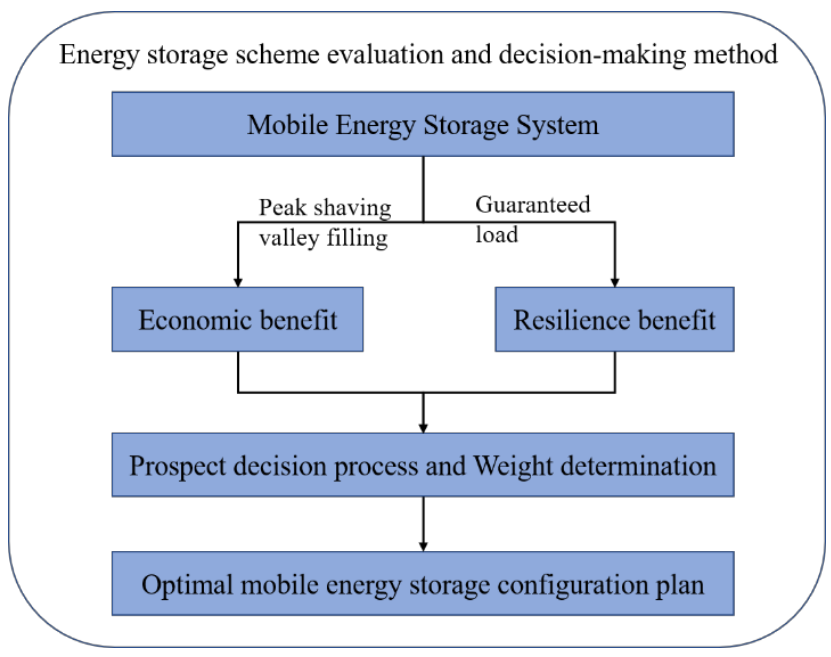

Figure 1. The overall assessment framework.

Taking a certain area as an example, in order to more effectively respond to extreme events, it is planned to select an optimal mobile energy storage configuration plan from 3 alternative points. The decision indexes $A_{1}$ $\mathrm{A}_{6}$ are the investment cost, the benefit from the participation of energy storage in normal operation, proportion of isolated nodes, fault recovery time, the switch operation times and the loss of power load. Based on historical experience and local conditions, decision makers make an evaluation of each attribute, as shown in Table 1.

Table1. Energy storage installation scheme evaluation matrix.

\begin{tabular}{|c|c|c|c|c|c|c|}
\hline & $\mathbf{A}_{\mathbf{1}}$ & $\mathbf{A}_{\mathbf{2}}$ & $\mathbf{A}_{\mathbf{3}}$ & $\mathbf{A}_{\mathbf{4}}$ & $\mathbf{A}_{\mathbf{5}}$ & $\mathbf{A}_{\mathbf{6}}$ \\
\hline $\mathbf{N}_{\mathbf{1}}$ & 79.97 & 31.61 & 0.31 & 3.15 & 3 & 54.79 \\
\hline
\end{tabular}




\begin{tabular}{|l|l|l|l|l|l|l|}
\hline $\mathbf{N}_{\mathbf{2}}$ & 150.32 & 59.52 & 0.42 & 6.04 & 2 & 62.03 \\
\hline $\mathbf{N}_{\mathbf{3}}$ & 171.73 & 67.99 & 0.47 & 5.73 & 2 & 64.17 \\
\hline
\end{tabular}

According the above formulas, calculate the schemes prospect value matrix and the result is shown in Table 2.

Table2. The prospect value matrix.

\begin{tabular}{|c|c|c|c|c|c|c|}
\hline & $\mathbf{A}_{\mathbf{1}}$ & $\mathbf{A}_{\mathbf{2}}$ & $\mathbf{A}_{\mathbf{3}}$ & $\mathbf{A}_{\mathbf{4}}$ & $\mathbf{A}_{\mathbf{5}}$ & $\mathbf{A}_{\mathbf{6}}$ \\
\hline $\mathbf{N}_{\mathbf{1}}$ & -1.12 & 0.5 & -1.13 & -1.12 & 0.5 & 0.5 \\
\hline $\mathbf{N}_{\mathbf{2}}$ & 0.08 & -0.75 & -0.48 & -0.04 & -1.01 & -0.76 \\
\hline $\mathbf{N}_{\mathbf{3}}$ & 0.51 & -1.1 & 0.52 & 0.294 & -1.12 & -1.13 \\
\hline
\end{tabular}

Calculate the decision weights $\omega 1=0.2467, \omega 2=$ $0.1126, \omega 3=0.120, \omega 4=0.0122, \omega 5=0.1558, \omega 6=$ 0.3373 . Then calculate the comprehensive prospect value $\mathrm{V}_{1}=-0.4689, \mathrm{~V}_{2}=-0.3512, \mathrm{~V}_{3}=-0.1586$. Therefore, the optimal sorting scheme can be obtained as $\mathrm{N}_{3}$.

\section{Conclusion}

Based on the prospect theory, this paper considers the psychological behaviour of decision-makers, and proposes a decision-making method for energy storage location and capacity. The different parameters in the value function of the prospect theory and the decision weight function indicate the different attitudes of decision makers to gain and risk. In decision-making based on prospect theory, decision makers adopt different parameters and different reference points in the value function and decision weight function according to their own preferences, and the prospect values of the obtained schemes may be different, and the ranking results may also be different.

On the one hand, evaluate the plan by establishing an indicator system that comprehensively considers the recovery capacity and economics. On the other hand, using the value function in the prospect theory and the attribute importance index based on the relative closeness to calculate the comprehensive value of the scheme, considering the risk preference of the decision maker in the face of gains and losses, the evaluation results are more in line with the psychological behaviour of the decision makers. Through the analysis of calculation examples, the rationality and feasibility of the method applied to the problem of energy storage site selection and capacity determination are verified. The method proposed in this paper has certain reference value for the research on the selection and evaluation of energy storage planning schemes.

\section{References}

1. Yang Huoming. Optimization method of energy storage system location and capacity considering the resilience of distribution network[D]. Shanghai Jiaotong University, 2018.

2. Gao Peng. Research on fault recovery method of active distribution network under extreme weather[D]. Yanshan University, 2019.

3. LEI Shunbo, WANG Jianhui, CHEN Chenl. Mobile emergency generator pre-positioning and real-time allocation for resilient response to natural disasters[J]. IEEE Transactions on Smart Grid. 2016,9(3):20302041.

4. LEI Shunbo, CHEN Chen, HUI Zhou. Routing and scheduling of mobile power sources for distribution system resilience enhancement[J]. IEEE Transactions on Smart Grid,2019,10(5):5650-5662.

5. Tversky K A. Prospect Theory: An Analysis of Decision under Risk[J]. Econometrica, 1979, 47(2):263-291.

6. Yongwei Yang. Distance-based multi-attribute decision-making method on improved situational fuzzy sets[J]. Fuzzy Systems and Mathematics, 2021, 35(02):107-114.

7. Hu Jun-hua and Zhou Yi-wen. Prospect theory based multi-criteria decision making method[C]. 2009 Chinese Control and Decision Conference, 2009,2930-2934.

8. Jiefang Liu, Sifeng Liu and Zhigeng Fang, Storage reliability evaluation model based on grey target theory combined with prospect theory[C].2015 IEEE International Conference on Grey Systems and Intelligent Services (GSIS), 2015, 374-377.

9. J. Yang, B. Yuan, F. Zhang, F. Luan, G. Lu and Z. Xu, Research on the Application Prospect of Energy Storage Technology in Energy Internet[C]. 2019 IEEE 3rd International Electrical and Energy Conference (CIEEC), 2019,1608-1612.

10. Liuxin Chen, Nanfang Luo. Pythagorean fuzzy multicriteria decision-making based on prospect theory [J]. Systems Engineering-Theory and Practice. 2020,40(03):726-735. 